\title{
Generalized Instructional Control and the Production of Broadly Applicable Relational Responding
}

\author{
Barry Lowenkron and Vicki Colvin \\ California State University, Los Angeles
}

\begin{abstract}
Two experiments examined the performance of preschool children in tasks requiring the generalized matching of faces to faces and names to faces under the control of instructional stimuli (background color) that specified the basis by which faces were to be matched on a given trial. The children first learned to recite all the names, and to select all the faces, in a fixed order (the forward order). They then learned to select the faces in response to their spoken names. When the faces appeared on a white background, subjects selected the face named. On a gray background, they selected the face whose name was next forward. Subsequently, over a series of tests, when subjects were presented with novel, but similar stimuli with the same names, and with completely novel stimuli with novel names, control by the white and gray background colors generalized. In the second experiment, on trials with the gray background, when the face bearing the next-forward relation was not present, the children learned to select an appropriate substitute (two faces forward). This performance also generalized to novel stimuli. Together with earlier findings, these data suggest that widely generalized relational matching performances may arise because the labels for these relations are generically and metaphorically extended tacts.
\end{abstract}

If subjects are instructed to select stimuli bearing a specific relation to a sample, and they understand the instruction, they would be expected to continue to select in accord with the instructed relation even when presented with novel stimuli. This generalized relational matching could be based on spatial dimensions such as length, area, and distance, wherein subjects are instructed to find stimuli that were longer, shorter, bigger, smaller, nearer or further than a sample, but it would also be expected with nonspatial relations such as order (before/ after), and numerosity (more/less).

Generalized relational matching may also occur where the instruction applies

This research was supported by a grant (HD 23123) from the National Institute of Child Health and Human Development. Our appreciation to Pam Kisor, Director of the Anna Bing Arnold Children Center, for her help and cooperation in coordinating subject participation. Portions of this research were conducted by the second author, under the guidance of the first, in fulfillment of the requirements for the Masters of Science degree in the Applied Behavior Analysis Option in Psychology at CSULA. Reprints may be obtained from Barry Lowenkron, Dept. of Psychology, California State University, Los Angeles, Los Angeles, CA 90032. across a variety of different dimensions. Thus, the relation lower can be applied to the intensity, value, strength, or pitch of various stimuli. And so, if subjects are told to select stimuli lower than the sample, their generalization to selections of the lower pitch, value, strength, or intensity would not be unexpected.

In these examples the instructed relations transcend the individual instances. Almost certainly it is for this reason, this lack of attachment to specific instances, that the sources of generalized relational matching tend to be seen to exist not within the stimuli selected (Skinner, 1969), but rather as an entity, concept, or schema (Bruner, Olver \& Greenfield, 1966; Inhelder \& Piaget, 1964), stored within the subject. As a result, the appearance of generalized relational matching is commonly viewed as an expression of relations stored, grasped, understood, or otherwise comprehended by the subject; and the instructions that specify these relations are viewed as names or labels that evoke these stored relations. 
For the behavior analyst, the problem these reifications present is self-evident; it prompts the question of how to characterize generalized relational matching without according the relations ontological status. In one such account (Sidman, 1986), instructional control is seen as a property of the 5-term contingency. In this case, instructional stimuli modify the effect of the sample in evoking a selection response to the appropriate comparison stimulus as a result of reinforced practice for such selections.

Such an account, however, encounters serious difficulties in explaining any kind of generalized relational matching with novel stimuli, because it must resort to some kind of transpositional responding along the physical dimensions of the stimuli (Spence, 1937). Given the restriction of this phenomenon to physical dimensions, and the demonstrated limitations of transposition (i.e., the distance effect) (Kendler, 1950), such an account is not convincing to begin with, becomes less so when the transposition must come under instructional control, and looses all applicability when applied to stimuli which do not vary on continuous dimensions such as faces and names.

An account that is appropriate to stimuli of this latter sort is offered by Hayes (1991) and by Steele and Hayes (1991). They suggest that generalized relation matching involves arbitrarily applied relational responding, based on relational frames that arise as the product of a particular history of training in language-capable subjects. The nature of this history has, however, not been clearly delineated

There is another possibility: one that does offer an explicit account of generalized relation matching. The purpose of the research reported here is to further develop this account. In an earlier study, Lowenkron (1989) identified a set of component responses that supported generalized relational matching under instructional control. In this study, young children were trained to select line lengths longer than, shorter than, or identical to a sample line; depending on the instruc- tional control exerted by the background screen color. As the components of this performance were trained, their cumulative effect on generalization was examined.

The performance involved a delayedmatching task. Children were first taught to respond to horizontal line lengths by setting a plastic compass to fit the ends of the lines. The children were also trained to subsequently transform the compass settings as a function of the screen color. On green, they made the compass setting one size larger, on blue, they left it unchanged, and on red, they made the setting one size smaller. Subjects were also trained to then select a line from a set of comparisons by finding one that fit the current compass setting without further changing the setting.

Generalization was then measured with two sets of novel stimuli. The first set contained rectangles of differing widths. This set was expected to allow for primary stimulus generalization from the horizontal line lengths trained initially, and thereby allow generalized matching. That was found. The second set contained stimuli that were all of the same size, but each stimulus contained a pair of dots separated by different distances. In this case, accurate matching did not appear. However, after the subjects learned to set the compass to the distance between dots, generalized instructional control over the selection of stimuli with dots nearer than, further than, or the same as the dot-pair in the sample, appeared immediately.

On some trials in these tasks, the comparison specified by the current sample and background color was not presented. Under these conditions subjects were taught to make a second transformation of the compass setting, still under the control of the background color, and find a comparison appropriate to the new compass setting. The comparison selected thus bore a constant relation to the sample even if it was not the comparison initially specified by the sample and the instructional stimulus. This constant-relation matching was also found to generalize across stimulus sets and instructional stimulus conditions. 
Thus, a highly generalized relational matching performance was produced.

Several aspects of the stimulus control developed in these performances deserve scrutiny. First, the mechanism by which comparisons were selected. As described earlier, the compass settings did not merely serve as $\mathrm{SD}_{\mathrm{S}}$ for a specific comparison selection, but entered into a more complex form of stimulus control over comparison selection. Thus, by requiring the children to select the comparison that (a) fit the current compass setting while (b) maintaining that setting unchanged, comparison selection responses were placed under the joint control of two sources of stimulus control. Previous studies involving a variety of response topographies have shown that bringing comparison selection under this form of stimulus control consistently produced generalized identity matching (Lowenkron, 1988) and generalized relational matching with novel stimuli as soon as accurate tacts for these stimuli were acquired (Lowenkron, 1984).

The second aspect to consider is the means by which relations between sample and comparison stimuli were controlled: they were trained as simple discriminated operants. The colors red, blue and green respectively specified the relations larger, same and smaller only in the sense that they controlled responses that transformed the compass setting to one size larger, left it unchanged, or made it one size smaller. Similarly, the failure to find the comparison specified by the sample produced constant-relation matching only through its control over the transformational response. Since none of these features depended on any characteristics particular to the stimuli being selected, the transformational responses, and their controlling instructional stimuli, generalized across all stimulus sets - thereby supplying the basis for generalized instructional control.

Probably the most pressing issue these data raise concerns the validity of using overt mediating behavior to simulate ordinary behavior. As a step toward answering this question, the current study replicated features of the earlier study, (Lowenkron,
1989) but used vocal, rather than physical, mediating behavior. To do this, nurseryschool children were trained to tact each face in a set with its name, and also to recite the names in a fixed order (i.e., as an intraverbal). They also learned to select the faces in response to spoken names as a function of the current screen color. On a gray background, they selected the face whose name was next forward from the sample. On a white screen they selected the face named as the sample. On some forward-matching trials, the named face did not appear, and here the subject had to select a face two names forward. Thus, the transformational responses that produced relational matching were brought under the control of two stimuli: background color and the absence of a specified stimulus (Lowenkron, 1992). The conditions under which this relational matching performance generalized to novel stimuli, so as to become broadly applicable, were then examined.

\section{EXPERIMENT 1}

\section{Subjects}

\section{METHOD}

One boy (SR) and 3 girls (AG, BB, JM) from the university day-care center served as subjects because children of this age were assumed to be less likely to have acquired relevant skills preexperimentally. All subjects were between 4-4 and 5-0 years old with a mean age of 4-6 years.

\section{Apparatus and Setting}

Apparatus. Stimuli were presented by a Commodore $128 \mathrm{D}^{\star}$ computer on a $45 \mathrm{~cm}$ Amdek $\mathrm{II}^{\oplus}$ color monitor. The monitorscreen locations touched on each trial were measured by a touch-sensitive screen (Personal Touch Corp. IBM ${ }^{\star}$ analog model) through an interface modified for the computer.

Setting. Sessions were run in a small, quiet room at the day-care center. The child sat at a table $59 \mathrm{~cm}$ high, facing the monitor. The experimenter sat to the right of the child in front of the computer keyboard. An observer sat behind the child. 


\section{Stimuli and Consequences}

Stimuli. The training-set stimuli consisted of the names and faces of five characters (Figure 1, panel A). In different parts of training, the characters were either displayed on the monitor screen or drawn in black on separate 7.5 by $13 \mathrm{~cm}$ cards. The stimuli of Transfer Set 1 consisted of the same names with the five characters shown in a full-body view. Transfer Set 2 contained five new characters and names. In all cases the characters measured $4.0 \mathrm{~cm}$ high by $3.5 \mathrm{~cm}$ wide on the monitor screen.

During the auditory-visual matching phases of the procedure, each trial began when a $2.0 \mathrm{~cm}$ black square appeared at the center of the monitor screen and a vocal sample, the character's name, was said once by the experimenter. During visual-visual matching, a character appeared as the sample. On both types of trials, when the subject touched the sample (black square or character) it was removed and replaced immediately (zero-delay matching) by four comparisons selected from the same stimulus set as the sample, with one comparison in each corner of the screen. Where subjects did not select a comparison within approximately $10 \mathrm{~s}$, they were prompted to "pick one."

Baseline and test blocks. Both baseline and test blocks consisted of 12 matching trials. Baseline blocks contained 12 trials with stimuli from the training set. Each training-set character appeared on at least two trials as the sample. Test blocks contained four trials with the training-set stimuli (Trials 1, 4, 8 and 10), interspersed with eight trials with Transfer Set (1 or 2) stimuli. Each transferset character appeared at least once as a sample.

Consequences. In both kinds of blocks, reinforcement was only available for correct responses on Trials 1, 4, 8, and 10 (the consequated trials). Responses during the other eight trials within each block had no immediate consequences (but see below) except to produce the beginning of the next trial. Incorrect selections on the consequated trials resulted in a 3-s screen blackout followed immediately by the next trial.

In both kinds of blocks, the first correct selection on a consequated trial was followed by a 2-s tone, while the Sesame Street $^{\star}$ character Big Bird ${ }^{\star}$ appeared at the location of the correct comparison. Then the screen cleared to a white background, and a string of 12 colored circles, or "cookies," appeared from left to right across the upper portion of the screen. A little boy appeared to the right of the rightmost cookie. Touching the screen at the location of the boy caused him to move to the left and pick up one cookie.

Subsequently, after correct selections on consequated trials, the string of remaining cookies was presented and the boy, when touched, picked up one cookie for the correct response on that trial. In addition, the boy remained on the screen to be touched again to pick up one cookie for each correct selection made on an unreinforced trial $(2$, $3,5,6,7,9,11,12)$ since the last consequated trial. To collect cookies owed for correct selections on trials 11 and 12, and because 12 correct selections were required to gain all the cookies, additional trials were presented by repeating the block until all the cookies had been collected.

This procedure, though complex, had certain advantages: On baseline blocks, subjects learned to respond without continuous reinforcement, and on tests, despite the fact that no response on a trial with transfer-set stimuli was reinforced, the overall reinforcement rate was correlated with the rate of accurate selections. Generalization was thus measured without immediate reinforcement.

After the 12th cookie was picked up, the procedure was ended, and the subject chose a sticker from among those lying on the table. On tests, if this occurred before the 24th trial, the appropriate block was presented again from the beginning. Thus, in test blocks, where trials of different types were interspersed, this affected the number of trials of each type that a subject was actually exposed to. The exact composition of the trial types given each subject is presented in their data.

Training criteria. Sessions were conducted three times per week and lasted an average of 30 minutes. The standard train- 


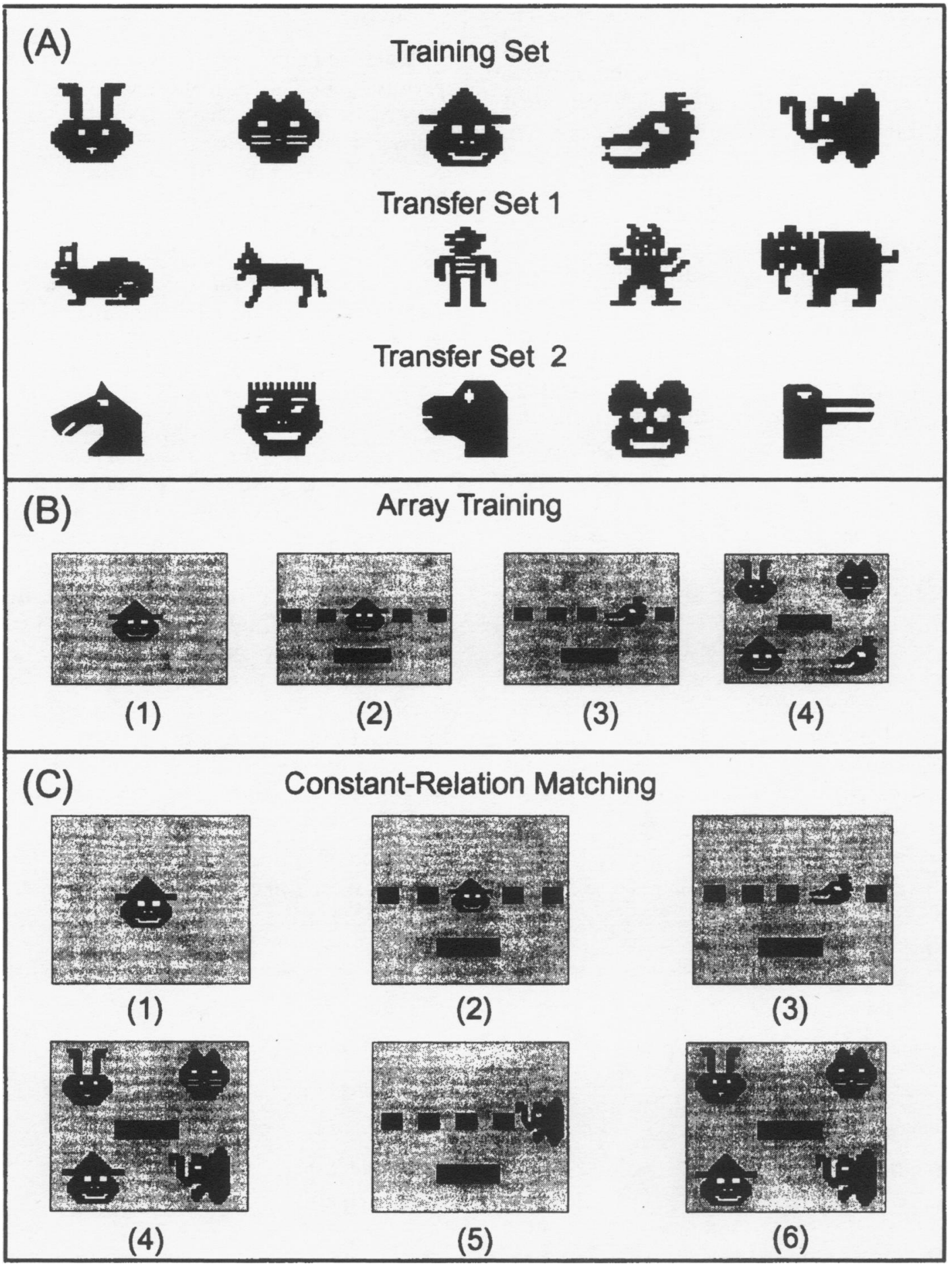

Fig. 1. Stimulus sets and matching tasks. (A) Stimulus sets for training and generalization (Transfer Sets 1 and 2 ). The shapes in the training set and Transfer Set 1 are named Bob, Carrie, Claude, Doug and Ella. In Transfer Set 2 they are Dan, Tom, Pete, Mike and Joe. The stimuli in all sets are shown (left to right) in the forward order. (B) Array training. (C) A constant-relation matching trial. The gray background specifies the forward-order matching relation. (1) Sample presentation. (2) The transformation array as it first appears given Claude was the sample. (3) The array after the subject has correctly transformed to Doug. Subsequently pressing the rectangle below the array produces (4) the comparisons. Upon failing to locate Doug, the subject presses the center rectangle to reproduce the array for a second transformation. (5) The array after a second transformation is made. (6) Subsequently, pressing the rectangle produces the comparisons. They contain the comparison (Ella) specified by the second transformation. 
ing criterion required 11 correct matches in one baseline block (12 trials). In training sessions, previously taught behavior was reviewed and, where necessary, retrained to this criterion before new behavior was trained.

Test sessions contained no more than four blocks. The first block was always a baseline block. If the standard training criterion was not reached during the first or second presentation of this block, two more baseline blocks were provided for practice, and the session ended after approximately 48 trials. If the standard training criterion was achieved within the first two blocks, two runs with an appropriate test block were presented to provide a minimum of 24 test trials: eight with the training set, and 16 with the transfer set stimuli. All touch-screen inputs, and the stimuli present at the locations touched, were recorded by the computer.

Observer reliability. On the first test, subject's verbalizations were recorded by both the observer and the experimenter. Agreement was found be $100 \%$, and so in subsequent tests the subjects' verbalizations were recorded by the observer alone.

\section{Procedure}

A detailed summary of the procedures is provided in Table 1 . Subjects were first taught to tact the five faces. They then learned to recite these names (an intraverbal) and to select the faces, in a fixed order. Next, in an auditory-visual matching task, the experimenter provided spoken names as samples, and the children were taught to select from four faces. When the screen color was white, they selected a face with the same name as the sample. When the screen was gray, they selected the face next-forward in the fixed order. The capacity of this performance to generalize was then measured over a series of tests. The first two tests measured generalization of the performance to similar stimuli before and after subjects were taught to tact the faces. The next test measured generalization to non-similar (i.e., novel) stimuli after tacts for these stimuli were taught, and the last two tests looked at generalization of the performance to visual-visual matching where spoken the sample names replaced by visual stimuli (the faces).

Tact training. In this phase, subjects learned to tact the training-set characters.

Table 1

Training and testing procedures in experiment 1.

Procedure

Tact training

Forward transformation training

Instructional control training

Generalization Test 1

Tact training

Generalization Test 2

Tact training

Forward Transformation training

Generalization Test 3

Train matching with visual samples

Generalization Test 4

Generalization Test 5
Stimulus Set

Sample

Training Set

Training Set

Training Set

Transfer Set 1

spoken

Transfer Set 1

Transfer Set 1

spoken

Transfer Set 2

Transfer Set 2

Transfer Set 2

spoken

Training Set

Transfer Set 1

picture

Transfer Set 2

picture 
Initially Bob and Carrie were presented on the monitor screen, one at a time, with a white background. The experimenter said the name (i.e., Bob) and then prompted the subject to repeat it ("Who is this?"). The remaining characters were added to the pair, one at a time. Training continued until the subject correctly tacted all the characters four times consecutively.

Forward-transformation training. This procedure trained subjects to repeat the names of the training-set stimuli in the forward order by transferring from tact control to intraverbal control (Partington \& Bailey, 1993). The names, in order, were Bob, Carrie, Claude, Doug and Ella. To train subjects to emit the names in this order as an intraverbal, the experimenter laid out the 7.5 by $13 \mathrm{~cm}$ cards on the table with the faces in this order, left to right. The subject was then asked "Who's first?" at the first character, and "Who's after?" at each subsequent character, to prompt the subject to tact them. After all five characters were tacted, the cards were moved so that an increasing fraction of each face (except the first), was overlapped by the adjacent card, and the procedure was repeated. This procedure continued until the subjects could name all five characters with the faces hidden - presumably as an intraverbal.

The next procedure trained the subjects to select the faces in the correct order. All five faces were presented in random locations on the monitor screen: three across the top and two across the bottom, on a gray background. The cards were again laid out in the forward order on the table for the subject to refer to. The experimenter then prompted the subject (Who is first? Who is next?) to tact each character and touch the corresponding face on the monitor. After all five faces had been touched, they were redistributed to new positions on the screen. Over trials, the cards were again gradually overlapped and finally removed. Training continued until the subject correctly tacted and selected the faces on the screen in the correct order through five redistributions.

Each correct selection of a face was reinforced with $3 \mathrm{~s}$ of music from the computer and occasional verbal praise. Incorrect selections resulted in a 2 -s screen blackout; when the faces reappeared, the experimenter prompted "It was (last name emitted), who's after?"

Instructional control training. This phase taught subjects to match the training-set faces to spoken names (auditory-visual matching) under instructional control. Subjects were taught to select the character named as the sample (referent match) when the background was white, and to select the character whose name was nextforward from the sample when the screen was gray.

In the first stage of training, a face was presented at the center of the monitor screen with a gray background. When the experimenter prompted ("Who is that?"), the subject tacted the face and touched it to produce the comparisons. The experimenter then asked "Who's after?" to prompt the subject to (intraverbally) transform the sample tact by saying aloud the next name forward in the forward order (an intraverbal), and then select the comparison so tacted.

In the second stage, still with a gray background, a black square replaced the sample face (auditory-visual matching). Now the experimenter said the sample name for the square (i.e., "That's Bob.") The subject then repeated the name, touched the square to produce the comparisons, and the procedure continued as in the prior stage.

In the last stage, half of the trials contained a white background and half a gray background, presented irregularly. To ensure these background colors were discriminated, subjects were required to begin each trial by tacting the background color. When the subject accurately tacted the color, the experimenter said the sample's name. When the subject repeated the name and touched the black square, the comparisons appeared. On referent-matching trials (white background), the selection prompt asked "Where is (sample name)?" The subject had to repeat the name again, and then select the comparison it tacted. On forward-matching trials (gray back- 
ground) the prompt was "Who's after?" The subject had to provide the next name forward in the trained order, and then select the comparison it tacted. Correct selections were reinforced by music and stickers. Incorrect selections resulted in a 2-s screen black-out followed immediately by the next trial. Over subsequent trials, the prompts were faded to a 5-s delay and then omitted.

In the last stage of instructional-control training, the baseline block was repeatedly presented. Training continued until the standard training criterion with the baseline block was reached in two consecutive training sessions. This auditory-visual matching performance constituted the baseline for Test 1.

Test 1 . This test measured the generalization of auditory-visual referent and nextforward matching to Transfer Set 1 . In the next session, after again reaching the standard training criterion with a baseline block, two test blocks were presented. The number of trials of each type is indicated in the data for each subject (Figure 2).

Tact training for Transfer Set 1 . The tacttraining procedure described previously was applied to the characters of Transfer Set 1.

Test 2. The procedures described for Test 1 were repeated.

Tact training and forward-transformationtraining for Transfer Set 2. The tact training and forward-transformation training procedures were applied to Transfer Set 2 (Figure 1, panel A).

Test 3. The procedure described for Test 1 was repeated, but here the test blocks contained names and faces from Transfer Set 2 .

Train matching with visual samples. In the next session, the black squares and spoken samples were replaced by the corresponding faces of the training set. Both matching relations and their respective instructional stimuli, the gray and white backgrounds, were maintained, but in all subsequent tests and training procedures, the experimenter no longer provided the sample's name; rather, the subject had to tact the sample face and press it in order to pro- duce the comparisons. On all training-set trials (but not on test trials), a correct sample tact was required. If the wrong tact was given, the subject had to correct it for the trial to continue. This change from auditory-visual to visual-visual matching changed the referent matching performance into identity matching.

Training continued to the standard training criterion in two consecutive sessions. Behavior on this task constituted the baseline for Test 4.

Test 4 . In the next session, after the subject reached the standard training criterion with the baseline for Test 4 , two test sequences with Transfer Set 1 were presented to measure generalized identity and next-forward matching with visual samples. Thus, the first test trial here was the very first time the transfer-set faces ever appeared as samples.

Test 5 . In the next session, the procedures described for Test 4 were repeated, but with the stimuli of Transfer Set 2 replacing Transfer Set 1.

\section{Results AND Discussion}

Before Test 1, no tacts had been trained for the stimuli of Transfer Set 1, and so generalized matching with these stimuli depended on primary stimulus generalization based on the features they had in common with the training set. Despite this (Figure 2), in Test 1, subject BB showed complete transfer of instructional control, making appropriate selections on both referent and next-forward trials with Transfer Set 1. With JM, instructional control was maintained with the training set, even as the subject made referent matches on almost all of the referent matching and next-forward trials with the transfer set. The spoken names thus controlled comparison selection here, even though instructional control did not generalize. In contrast, subjects AG and SR, showed little generalized matching.

After the subjects had learned to emit the tacts for the transfer set stimuli, and generalization was retested in Test 2 , instructional control over the matching relations with Transfer Set 1 improved in subject JM. 


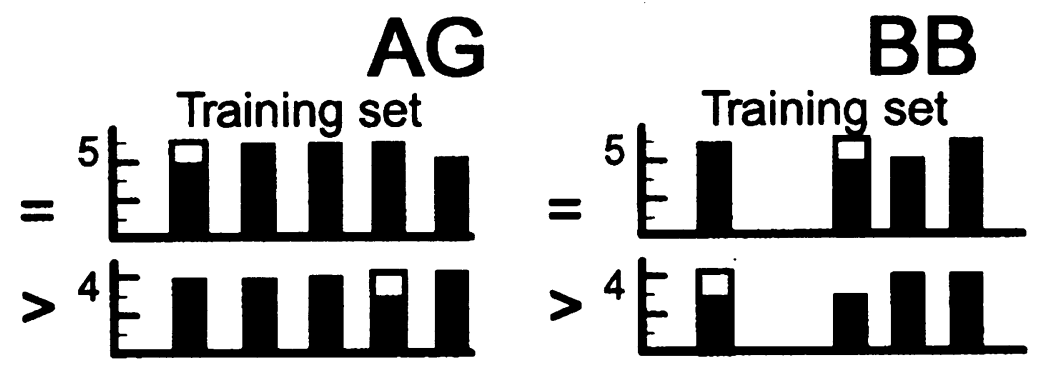

Transfer Set: $\begin{array}{lllll}1 & 1 & 2 & 1 & 2\end{array}$
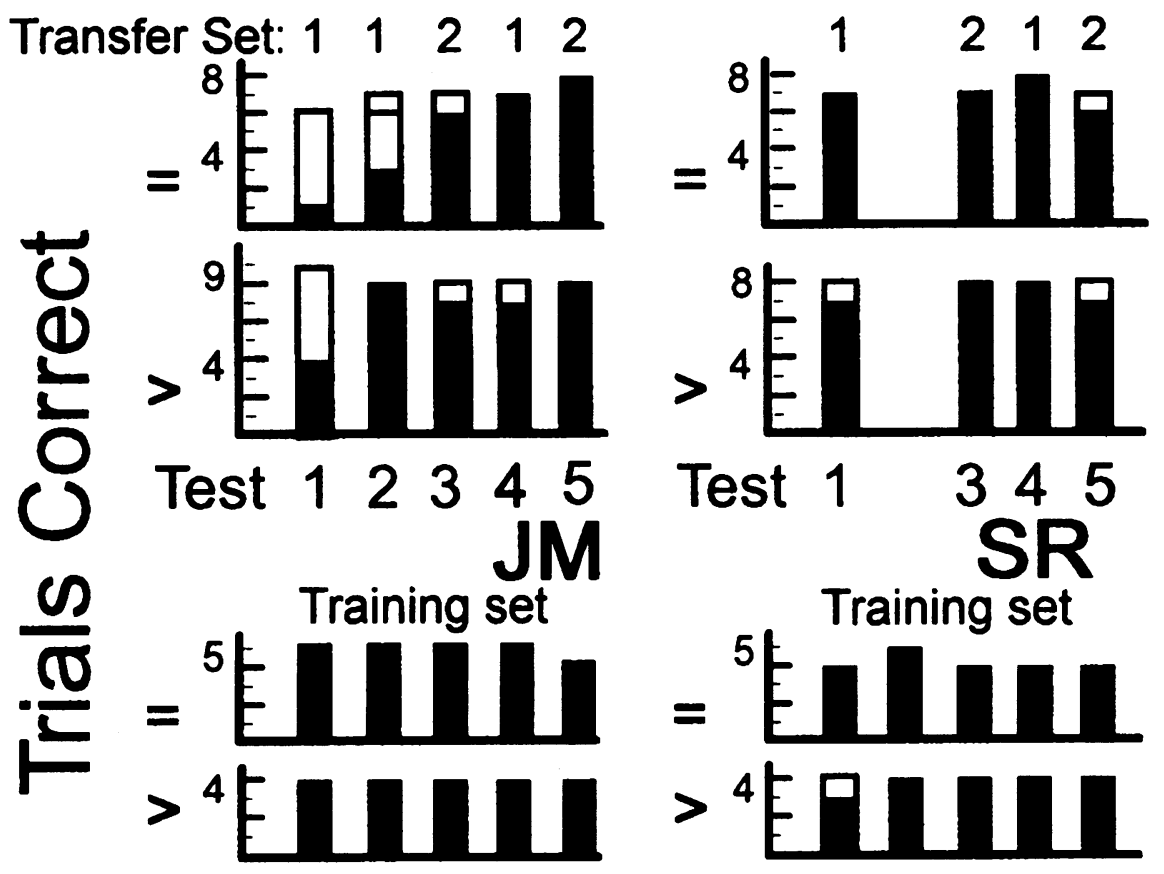

Transfer Set: $\begin{array}{lllll}1 & 1 & 2 & 1 & 2\end{array}$
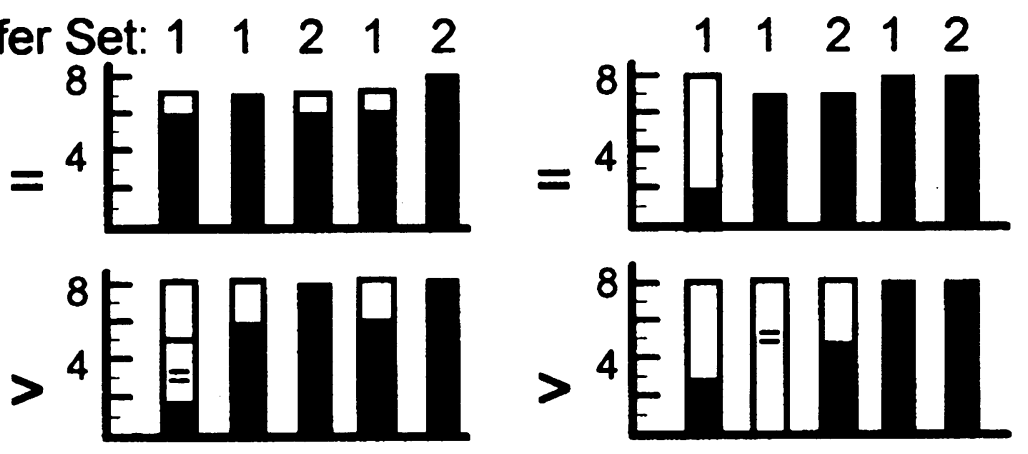

Test 12345

Test 12345

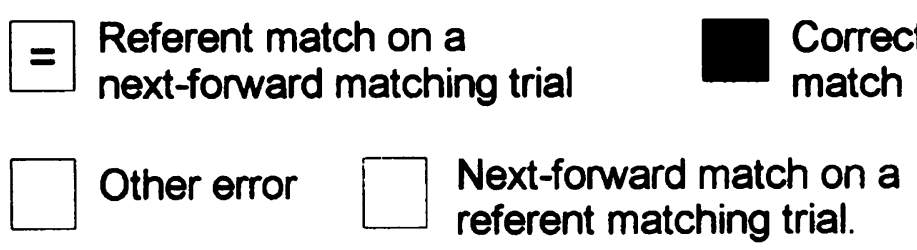

Fig. 2. Matching performance in Test 1,2 and 3. The hollow portions of columns indicate trials with incorrect matches. 
(Because of the high accuracy in Test 1, BB was not given Test 2.) In contrast, subject SR improved in referent matching, but like JM in Test 1, appeared to loose instructional control with the novel stimuli: making referent matches on all next-forward trials with Transfer Set 1 even while instructional control was maintained with the training set.

Since the samples were spoken words, these errors cannot be attributed to the subjects' reliance on the physical identity of samples and comparisons. Rather, it would appear that on these trials the subjects failed to transform the sample name to the next-forward name; selecting instead the comparison named by the experimenter.

This reversion to referent matching on the forward-matching generalization trials replicates data reported in two earlier studies (Lowenkron, 1984, 1989). In both of these studies, after the subjects learned to select various relations under instructional control, their performance often reverted to identity matching on trials where novel stimuli were introduced. Direct measures of the overt mediating responses trained in these studies revealed that these errors resulted from the subjects' omissions of transformational responses. These data thus suggest that in the current experiment as well, the loss of instructional control over relational matching in subjects SR and JM may have been the result of a loss of instructional control over a specific operant: the intraverbal transformational behavior.

With subject AG, instructional control on generalization trials also appears to have been lost in Test 2. But in contrast to SR and JM in Test 1 , this subject selected the next-forward comparison on almost all generalization trials; thereby illustrating in a different way that the sample stimuli and the instructional stimuli each had an independent role in comparison selection, and that they thus did not function as conditional stimuli acting as members of a higher-order contingency.

When the names and the forward-list order were trained with Transfer Set 2, high levels of generalized instructional control appeared in Test 3 despite the fact that these trials provided the first opportunity for the subjects to respond to the stimuli in this set by selecting them rather than by tacting them. This suggests that the loss of instructional control seen in Test 2 was due more to the introduction of the novel stimuli of Transfer Set 1 without warning than to any loss of stimulus control over selection. Apparently, with this novelty worn off, subjects were able to match accurately in Transfer Set 2 from the very first.

When visual samples were introduced in Test 4 with Transfer Set 1 , and again in Test 5 with Transfer Set 2, subjects provided accurate tacts for the sample stimuli and accurate matching continued without disruption. No subject (Figure 2) made more than three errors in either test. Certainly the simplest explanation would have it that the subject-provided tacts for the stimuli replaced the experimenter-supplied tacts.

Perhaps what is most interesting about behavior in Tests 2 through 5 is the illustration it provides of how an instructional stimulus can come to control different kinds of matching relations even as it maintains control over the same transformational response topographies. Thus, in Tests 2 and 3, a white screen controlled the selection of the comparison named by the sample, while in Tests 4 and 5 a white screen controlled selection of the comparison identical to the sample. Similarly, in Tests 2 and 3 a gray screen evoked selection of a comparison not named by the sample, while in Tests 4 and 5, the gray screen evoked selection of a comparison stimulus not identical the sample.

Taken across Tests 2-5, white might thus be said to serve as a label for the higherorder relation of equivalent to, while gray signified the relation not equivalent. Comparable performances were directly observed by Lowenkron (1989) where two instructional stimuli controlled overt transformational responses for the spatial relations shorter than, and longer than as well as for nearer, and further. The stimuli thus came to function as labels for the more general quantitative relations less and more. 


\section{EXPERIMENT 2}

The tasks used in the prior experiment may be described as simple matching because each sample specified a single comparison. Prior research (Lowenkron, 1989) has demonstrated, however, that the performances described here have the capacity to generalize to more complex tasks.

One such task is constant-relation matching. In this task, on trials in which a comparison bearing a specified relation (i.e., next size larger) is not found, the subject must select, from among the comparisons available, the one closest to the specified relation (i.e., next available size larger). Thus the relation of the search target to the sample remains constant even if the target itself does not. Such a performance has a property typical of a goal-oriented behavior: in the face of the failure of a search, the subject changes the search target in a systematic fashion.

In this experiment, constant-relation matching is trained for the next-forward matching relation. If the properties of the behavior trained here parallel those trained in the prior study, it will produce a generalized performance in which subjects, under the control of instructional stimuli, select either an identity match, or a nextforward match. But on the latter type of trial, if the comparison next forward in the trained order is not found, subjects will change the target of their search to a comparison two steps forward from the sample. The next-forward transformation thus comes under two sources of control: background color, and the absence of the initially specified comparison (Lowenkron \& Colvin, 1992).

\section{MeTHOD}

\section{Subjects}

All of the subjects from Experiment 1 were retained and their training continued.

\section{Stimuli}

The transformation array. As illustrated in Figure 1 (panel B), after the sample was pressed, a row of five $1.5 \mathrm{~cm}$ black squares appeared across the center of the monitor as a transformation array. Each square represented the training-set character which held the corresponding position in the forward order (Figure 1, panel A). In addition, a 0.8 by $3.5 \mathrm{~cm}$ black rectangle appeared either below the array or at the center of the comparisons. Pressing this rectangle allowed subjects to toggle the view back and forth between the transformation array and the comparisons.

\section{Procedure}

A summary of the procedures is given in Table 2.

Array training. This procedure (Figure 1, panel B), taught subjects to transform samples in the forward order using the visual stimuli of the transformation array. Training was begun in the session following Test 5. A sample face was presented (panel B [1]). After the subject tacted and touched the sample, the transformation array appeared with the sample face at the location in the array that corresponded to its position in the forward order. Thus, in panel $B(2)$, the clown, which holds the third position in the forward order, now appears in the third position in the array.

On forward-matching trials (gray screen as shown here), the experimenter pointed to the square in the array to the right of the sample face and told the subject to press it. Once it was pressed, the sample face was replaced by a square and the training-set face of the next character in the forward order appeared in place of the square pressed (as shown in panel $B[3]$ ). The experimenter then asked "Who is it?" to prompt the subject to tact the newly visible character. Subjects were then prompted to press the rectangle below the array to view the comparisons (panel $B[4]$ ).

When the comparisons appeared, subjects were prompted to select the comparison last tacted. If the subject did not select a comparison, the experimenter asked "Who do you need?" to prompt the subject to select the last character tacted. If the subject could not remember who it was, he/she was prompted to touch the rectangle at the center of the comparisons. This 
Table 2

Training and testing procedures in experiment 2.

\begin{tabular}{llll}
\hline Procedure & Stimulus Set & Array & $\begin{array}{l}\text { Matching } \\
\text { Relations }\end{array}$ \\
\hline Array training & Training Set & operational & $=,>$ \\
Train constant-relation matching & Training Set & operational & $>,>>$ \\
Generalization Test 6 & Transfer Set 1 & operational & $=,>$, >> \\
Generalization Test 7 & Transfer Set 2 & operational & $=,>$, >> \\
Generalization Test 8 & Training Set & inoperative & $=,>,>>$ \\
Train matching with array inoperative & Training Set & inoperative & $=,>,>>$ \\
Generalization Test 9 & Transfer Set 1 & inoperative & $=,>,>>$ \\
Generalization Test 10 & Transfer Set 2 & inoperative & $=,>,>>$ \\
\hline
\end{tabular}

Note. Referent matching, next-forward matching and constant-relation matching are indicated by the symbols $=,>$, and $>>$ respectively.

caused the transformation array to reappear with the product of the last transformation still visible (i.e., panel $B[3]$ ). The subject could then press the bottom rectangle to toggle back to the comparisons and make a selection.

On identity-matching trials (white screen), when the array appeared, the subject was prompted to repeat the sample name and press the bottom rectangle so as to produce the comparisons without transforming the array. When the comparisons appeared, the experimenter asked "Where is (sample name)?" to prompt the subject to select the sample character - thus generating an identity match. Over subsequent trials the prompts in both conditions were faded to a 5-s delay and finally omitted. Training continued until the standard criterion was attained in two consecutive training sessions.

Training constant-relation matching. In this task, on some next-forward matching trials, the comparison (e.g., Doug in Figure 1, panel $\mathrm{C}[3]$ ) specified by the first transformation was not among the four available for the subject to select among (Figure 1, panel C[4]). Rather, the subject had to learn to repeat the transformation in order to select the second comparison forward, Ella.

Because these constant-relation matching trials were interspersed with simple next-forward matching trials, they could only be detected by the failure of a search to find the first comparison. Therefore, on these trials, after it was determined that the subject could not find the first comparison (by his answering "no" to the query "Is he there?"), the experimenter pointed to the central rectangle and told the subject to "Press it to go back" (to the array). At the transformation array, the experimenter said "Who do you want? Press here to see." and pointed to the square to the right of the currently visible character -- thereby prompting the subject to make a second transformation (Figure 1 panel $C[5]$ ). When the square was touched, it was replaced by the character corresponding to that position in the forward order while a square replaced the last character. The experimenter then prompted the subject to tact the newly visible character, press the bottom rectangle to return to the comparisons, and select one. Over subsequent trials, all prompts were faded to a 5-s delay and finally omitted.

Since selection did not wrap around the end of the list, on simple forward-matching trials Ella could not be the sample, and likewise on constant-relation trials neither Doug nor Ella were ever presented as samples.

In the final phase, training continued with a baseline block containing identity trials (white background) interspersed 
with simple forward and constant-relation forward-matching trials (both on a gray background) until the training criterion was attained. Initially, this performance provided the baseline for Test 6, but was repeated later to provide baselines for Tests 7 and 8.

Test 6 . In a subsequent session, after reaching the training criterion with the baseline for Test 6 , two test blocks were presented to measure the generalization of constant-relation matching to Transfer Set 1. Subjects were still required to correctly tact each training-set sample for the trial to proceed. Tacts for transfer-set samples were recorded, but a correct tact was not required for the trial to proceed. The composition of the trials in this test is indicated in the data.

Test 7. After subjects reviewed the tacts and the forward order for the stimuli in Transfer Set 2, followed by one block at the training criterion on the baseline for Test 6 , two test blocks with Transfer Set 2 were presented as Test 7 . Here the transformation array contained faces from Transfer Set 2.

Test 8. This test measured matching in the training set with the transforming function of the array made inoperative. After again completing one block at the training criterion on the baseline for Test 6 , the test began. Two more baseline blocks were presented. In these blocks, events in identity matching trials remained unchanged: Pressing the sample still produced the array with the sample face at the correct position, and subjects could still press the bottom rectangle to proceed to the comparisons. But on the simple forward matching, and on constant-relation matching trials, pressing a square in the array had no effect - the sample face in the array remained visible, and the next face forward did not appear.

In response to subjects puzzlement on the first few trials, the experimenter pointed to the correct square in the array and asked, "Who is that?" When subjects answered, whether correctly or not, the experimenter pointed to the bottom rectangle and told subjects to press it and thus display the comparisons. On constant-relation trials subjects could still toggle freely between the (inoperative) array and the comparisons.

Training matching with the array inoperative. In the next session, the performance measured in Test 8 was trained for use as a baseline. Subjects learned to substitute names for the pictures in the inoperative array. When a sample appeared, the subject tacted and touched it to produce the array. If needed, the experimenter then asked "What do you do?" to prompt the subject to press the square in the array one place to the right of the (visible) sample character and "Who is that? to prompt the subject to name the character (now presumably as an intraverbal, since they were no longer visible) whose square in the array had just been pressed. Pressing the rectangle again produced the comparisons.

On constant-relation trials, the subject could toggle back to the still-inoperative array, and make a second verbal transformation - pressing the square while saying the name of the next character forward (presumably as an intraverbal) - before selecting a comparison. This performance comprised the baseline for Test 9 and later for Test 10.

Test 9. In the next session, after reaching the standard training criterion with the above-described baseline, two test blocks measured the generalization of constantrelation matching to Transfer Set 1 with the array inoperative.

Test 10. In the next session, after subjects tacted the stimuli of Transfer Set 2 and rehearsed the forward transformation with them (both without error), the procedure described in Test 9 was repeated, except the test contained the stimuli of Transfer Set 2 instead of Transfer Set 1.

\section{Results AND Discussion}

Using visual samples and the transformation array allowed four components of the matching performance to be measured directly. The first component, the samplecoding response, was scored as correct if the subject correctly tacted the character appearing as the sample. This behavior is 
not reported for training-set trials, since subjects were required to tact samples correctly for the trial to proceed. Errors here were virtually non-existent.

The second and third components, the two transformations, were scored as follows. On constant-relation matching trials, the first transformation was scored as correct if the subject changed the array by pressing the square to the right of the sample before viewing the comparisons. The second transformation was scored as correct if, after viewing the comparisons, the subject returned to the array and pressed a square immediately to the right of the one pressed in the first transformation.

On a simple forward-matching trial, the first transformation was still scored as correct if the subject transformed correctly before viewing the comparisons. The second transformation was scored as correct if, after viewing the comparisons, the subject did not return to the array and press a square. On an identity trial, both transformations were scored as correct if the subject left the array unchanged throughout the trial. Where the array was inoperative, both the spoken transformations of the names and attempts to change the array were recorded.

The final component, comparison selection, was scored as correct if the comparison selected had the same name as the character specified by the most recent transformation; regardless of whether or not that transformation was itself correct.

Finally, an overall match was scored as correct, without regard to mediating behavior, if the comparison selected was correct given the sample and the current instructional stimulus. A correct match was thus partially redundant upon the correct performance of all components, but the effects of inaccurate performances on two or more components could cancel to produce a correct match.

After constant-relation matching was acquired, accuracy remained high with the introduction of Transfer Set 1 in Test 6 (Figure 3). The immediacy of this generalization indicates that subjects used the stimulus names as a basis for transforming and selecting. Since the array contained faces from the training set on both training and Transfer Set 1 trials, only pre-existing names, common to the corresponding stimuli in both sets, would allow subjects to match from the very first on trials where a transfer-set sample, a training-set array, and transfer-set comparisons appeared. The equally rapid generalization with the stimuli of Transfer Set 2 in Test 7 further attests to the generality of stimulus control over constant-relation matching; showing that it did not depend on any aspects unique to the training set or to their samenamed variants in Transfer Set 1.

The accurate comparison selection on constant-relation trials in both Tests 6 and 7 indicates a role for some form of generalized stimulus control exerted by the absence of specified comparisons (See Lowenkron \& Colvin [1992] for a discussion and demonstration of how the absence of a stimulus may control behavior.) Thus, although subjects had only been trained to return to the array and transform on trials with training-set stimuli, the performance appeared on trials in both Transfer Sets 1 and 2 with no direct reinforcement.

Since the absence of (or alternately, the failure to find) the specified comparison was the only common element in these trials, it seems self-evident that this event played a role in the rapid generalization of constant-relation matching. This generalization not only emulates a highly abstract performance, but by changing the search target on constant-relation trials, the performance appears goal-oriented. Subject sought the next available stimulus bearing a specified relation to the sample regardless of the particular physical characteristics of the stimulus itself.

The continued dependence of accurate matching on the mediating transformational behavior is illustrated by the fact that matching errors occurred on $75 \%$ of all trials containing a transformation error in Test $6,66 \%$ in Test $7,58 \%$ in Test 9 and $40 \%$ in Test 10 . Interestingly, on constantrelation matching trials there were no differences in the error rates for first or second transformations. 


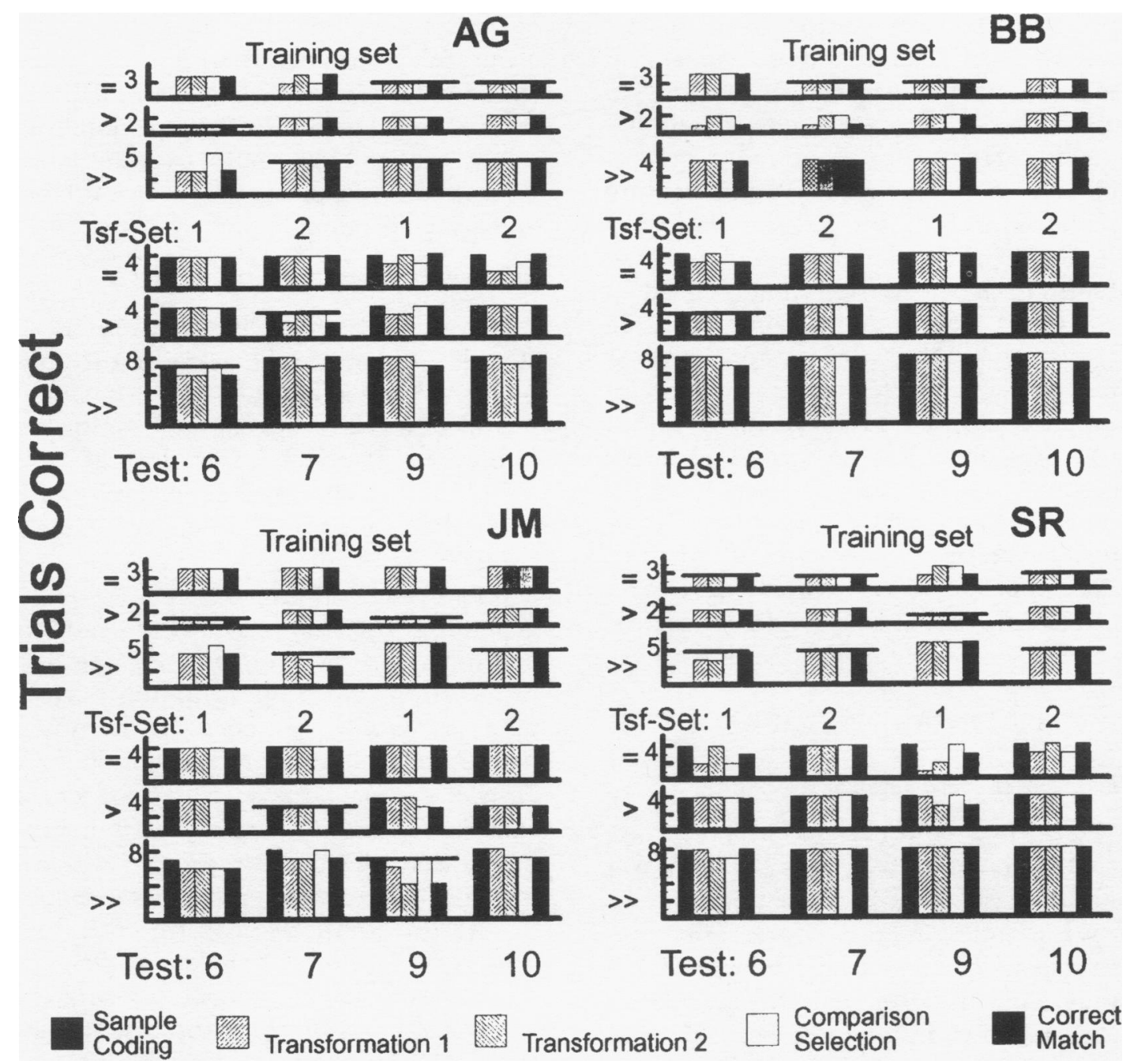

Fig. 3. Accuracy in performing each component behavior and in overall matching in identity $(=)$, simple relation (>) and constant relation (>>) matching with the training set and Transfer Set 1 in Tests 6 and 9 and with the training set and Transfer Set 2 in Tests 7 and 10. Horizontal bars indicate the number of trials of each type where it differs from the highest value shown on the ordinate.

The dependence of accurate matching on accurate transformations showed up in a different way in Test 8 . Thus, when the array was made inoperative in Test 8 , performance was completely disrupted, and matching accuracy fell to random levels in all subjects.

However, in subsequent training with the array inoperative, accurate matching was regained in all subjects by prompting on no more than 6 trials. This rapid switch back to vocal mediation suggests that the initial disruption in Test 8 was due more to the novelty of the event than to the loss of any crucial source of stimulus control in the array. Apparently, in Tests 6 and 7, subjects had let the changing faces of the array specify the name of the next comparison face to select. That is, they were tacting the faces that appeared as squares in the array were pressed. When this source of control was removed in Test 8 , after the initial disruption, subjects apparently reverted quickly to the intraverbal transformations they had used in Tests 1 through 5.

As illustrated in Figure 3, matching with the array inoperative generalized to Transfer Set 1 in Test 9. The transformation data shown here describe the accuracy of 
intraverbal transformations, that is, the accuracy of the name said as a square on the now inoperative array was pressed. No subject pressed more than one square that was incorrect for the name emitted. Subjects were thus able to transform by accurately pressing and naming the correct squares in the array without relying on the squares to change to faces when pressed.

In Test 10 , accurate matching with the array inoperative also generalized in Transfer Set 2 despite the fact that this performance required transformations with a sequence for which the array had never been inoperative. None the less, subjects immediately provided appropriate intraverbal transformations for the stimuli under appropriate instructional control on both simple and constant-relation matching trials.

\section{GENERAL DISCUSSION}

\section{The role of transformational responding}

Two facts suggest that the various matching relations seen here did not result from the sample, comparison, and instructional stimuli acting under conditional control as parts of a five-term contingency, but rather from the direct control exerted by instructional stimuli over transformational responding to the samples.

First, in Experiment 1, inappropriate referent matching (on the next-forward trials) and inappropriate next-forward matching (on referent trials) appeared with the introduction of novel stimuli even while the alternate matching relation with these stimuli was retained. That this was due to the loss of instructional control over the transformational response is suggested by the fact that these data replicate two earlier studies (Lowenkron 1984, 1989) where mediating responses were overt, and the loss of transformational responding with the introduction of novel stimuli was observed directly.

A second, and more direct form of support for the role of mediating transformational responses is provided in Experiment 2 by the data, cited earlier, illustrating the dependence of accurate matching on accu- rate transformational responses to the array. Taken together, these findings argue that in the performance trained here, instructional stimuli affected matching solely through their control over the transformational responses - both intraverbal and array-changing.

\section{The emergence of generalized relations}

The control exerted by the instructional stimuli was not specific to transformations with the trained stimuli: The intraverbal recital of a list of names for training-set stimuli (next-name forward), was first found to generalize to the physically similar transfer-set stimuli where the experimenter supplied the sample names (Test 1 and 2). Later, the performance was seen to generalize to a task in which the subjects had to supply the sample names (Test 4). In addition, the transformation performance was maintained with the novel names of the stimuli of Transfer Set 2 (in Tests 3 and 5), and later, with both sets of stimuli, in a task (constant-relation matching) in which the occasion for an additional transformation was supplied by the failure to find the comparison specified by the first transformation. The relation, after was thus instantiated in a number of different instances by a single transformational operant. Thus, consistencies in the subject's matching behavior across a variety of tasks, which might typically be ascribed to some conceptual relation possessed or known by the subject, could in fact be ascribed to the generalization of a single, broadly applicable, transformational response - in this case the intraverbal recitation of a list of names.

\section{The emergence of general labels and broadly applicable relations}

Beyond the single transformation, a broader level of generalized application appears when a collection of different transformational responses come under the control of a common instructional stimulus. Thus, in the present case, referential and identity matching were controlled by a white screen color. In terms of over-all matching, white might be said to have 
served as a name, label or signal for the conceptual relation same as just as the gray background was the label for next.

But if these subjects had also been trained in the tasks described in Lowenkron (1989), such that the white screen color also controlled the selection of line lengths, rectangle widths and dot distances identical to the sample, then white could now be said to serve as a label for the broadly applicable relations same as or equivalent to across a wider variety of dimensions and tasks. Similarly, if in addition to the forward transformation trained here, gray were made to control the nextlarger transformation in the selection of lengths, widths and distances, then gray could be said to be a label for the broad relation, roughly, greater or more than. In essence then, relations become more general as their labels become more broadly applicable, i.e., as more transformations, each within its own context, come under the control of a common instructional stimulus.

As to the process by which additional transformations come under the control of a common instructional stimulus, conceptual analysis suggests that it could arise from the generic extension of tacts (Skinner, 1957, p. 91). Consider for example, the relation lower. The tact low may be applied to the pitch of a note, the amplitude of a sound, the price of an item, or the location of something. Skinner (1957) suggests that these varied usages of the word low are the result of generic and metaphoric extension (i.e., generalization along common, relevant or irrelevant elements of the stimuli). In each case, a different response would be required to transform a stimulus to conform to this label, i.e., to lower the property (pitch, amplitude, price or location) named, but as these different transformational responses are acquired and brought under control of the common instructional stimulus lower, it appears that the relation lower is being applied in an increasingly broader manner.

Thus, through the processes of tact extension, a seemingly arbitrary collection of diverse transformations may come under the control of a single instructional stimulus. When this stimulus is subsequently applied to a particular matching task, it evokes the appropriate transformation response; thereby allowing the selection of stimuli bearing the relation to the sample specified by the instruction. On this view a set of diverse, broadly applicable relational matching performances controlled by a common label, may be accounted for in terms of the interaction of simple operants.

\section{REFERENCES}

Bruner, J.S., Olver, R. R., \& Greenfield, P. M. (1966) Studies in cognitive growth. New York: John Wiley.

Hayes, S. E. (1991). A relational control theory of stimulus equivalence. In L. J. Hayes \& P. N. Chase (Eds.), Dialogues on verbal behavior (pp. 19-40). Reno, NV: Context Press.

Inhelder, B., \& Piaget, J. (1964). The early growth of logic in the child. New York: Norton.

Kendler, T. S. (1950). An experimental investigation of transposition as a function of the difference between training and test stimuli. Journal of Experimental Psychology, 40, 552-562.

Lowenkron, B. (1984). Coding responses and the generalization of matching-to-sample in children. Journal of the Experimental Analysis of Behavior, 42, 1-18.

Lowenkron, B. (1988). Generalization of delayed identity matching in retarded children. Journal of the Experimental Analysis of Behavior, 50, 163-172.

Lowenkron, B. (1989). Instructional control of generalized relational matching to sample in children. Journal of the Experimental Analysis of Behavior, 52, 293-309.

Lowenkron, B., \& Colvin, V. (1992). Joint control and generalized nonidentity matching: Saying when something is not. The Analysis of Verbal Behavior, 10, 1-10.

Partington, J. W., \& Bailey, J. S. (1993). Teaching intraverbal behavior to preschool children. The Analysis of Verbal Behavior, 11, 9-18.

Sidman, M. (1986). Functional analysis of emergent verbal classes. In T. Thompson \& M. D. Zeiler (Eds.), Analysis and integration of behavioral units (pp. 213-245). Hillsdale NJ: Lawrence Erlbaum Associates Publishers.

Skinner, B. F. (1957). Verbal behavior. New York: Appleton-Century-Crofts.

Skinner, B.F. (1969). The inside story. In B. F. Skinner, (Ed.), Contingencies of reinforcement: A theoretical analysis (pp. 269-297). New York: AppletonCentury-Crofts.

Spence, K. W. (1937). The differential response in animals to stimuli varying within a single dimension. Psychological Review, 43, 427-449.

Steele, D., \& Hayes, S. C. (1991). Stimulus equivalence and arbitrarily applicable relational responding. Journal of the Experimental Analysis of Behavior, 56, 519-555. 